\title{
Cured primary hyperparathyroidism after fine-needle aspiration biopsy-induced parathyroid disappearance
}

\author{
Elda Kara1, Elisa Della Valle1, Sara De Vincentis', Vincenzo Rochira1,2 and Bruno Madeo² \\ 1 Unit of Endocrinology, Department of Biomedical, Metabolic and Neural Sciences, University of Modena and Reggio \\ Emilia, Modena, Italy and 2Unit of Endocrinology, Department of Internal Medicine, Endocrinology, Metabolism, and \\ Geriatrics, Azienda Ospedaliero-Universitaria di Modena, Ospedale Civile di Baggiovara, Modena, Italy
}

Correspondence should be addressed to B Madeo

Email

bruno.madeo@unimore.it

\section{Summary \\ Spontaneous or fine-needle aspiration (FNAB)-induced remission of primary hyperparathyroidism (PHPT) may occur, especially for cystic lesions. However, the disease generally relapses over a short time period. We present a case of PHPT due to an enlarged hyperfunctioning parathyroid that underwent long-term (almost 9 years) clinical and ultrasonographic remission after the disappearance of the lesion following ultrasound (US)-assisted FNAB. A 67-year-old woman with PHPT underwent biochemical and US examinations that confirmed the diagnosis and showed a lesion suggestive for parathyroid adenoma or hyperplasia. US-FNAB of the lesion confirmed its parathyroid nature by means of elevated levels of parathyroid hormone within the needle washing fluid. At the second visit, the patient referred slight neck swelling that resolved spontaneously in the days after the US-FNAB. At subsequent follow-up, the enlarged parathyroid was not found; it was visible neither with US nor with magnetic resonance imaging. Biochemical remission persists after 9 years. This is the first reported case of cure of PHPT after US-FNAB performed on a hyperfunctioning parathyroid resulting in its complete disappearance over a period of 9 years of negative biochemical and ultrasonographic follow-up.}

\section{Learning points:}

- Spontaneous or fine-needle aspiration-induced remission of primary hyperparathyroidism can occur.

- Both circumstances may present disease relapse over a variable time period, but definite remission is also possible even though long-term periodic follow-up should be performed.

- Parathyroid damage should be ruled out in case of neck symptomatology after parathyroid fine-needle aspiration or spontaneous symptomatology in patients with history of primary hyperparathyroidism.

\section{Background}

Primary hyperparathyroidism (PHPT) is the most common cause of hypercalcemia and is often caused by a single adenoma (85\%) or four-gland hyperplasia (15\%) (1). Functional cysts are less common etiologies while parathyroid carcinoma is a very rare cause of PHPT (1). Parathyroid ultrasonography (US) and sestamibi scan represent the first steps for parathyroid localization (2). High-resolution US is safe, rapid and easy to perform in skilled hands (3). US, however, is operator dependent and concomitant thyroid nodules may cause false-negative results (3). Furthermore, parathyroid cytology has very low diagnostic value even when FNAB is coupled to US (US-FNAB) $(2$, 4). For these reasons, in clinical practice, the measurement of intact PTH within the washing fluid (wPTH) obtained by US-FNAB, increases the US positivepredictive value for the detection of parathyroid lesions and is recommended as an ancillary study in order to distinguish parathyroid from thyroid lesions on US-guided $\operatorname{FNAB}(4,5)$.

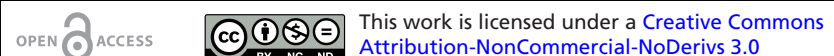
Unported License.
} 
At present, parathyroidectomy is the treatment of choice and the only definitive one for symptomatic PHPT (6). However, in some particular circumstances, other invasive procedures or pharmacological treatments can be indicated, especially in case of contraindications to surgery, surgical failure or no current criteria for surgical treatment (6).

We present a case of PHPT due to an enlarged parathyroid that underwent persistent long-term biochemical and ultrasonographic remission (almost nine years) after the US-FNAB-induced disappearance of the parathyroid lesion. To our knowledge, this is the first case of PHPT with such a long remission and essentially a complete cure of the disease.

\section{Case presentation}

A 67-year-old woman presented at the outpatient clinic of the Unit of Endocrinology of Modena on December 2008 because of mild hypercalcemia (calcemia $11.3 \mathrm{mg} / \mathrm{dL}$, normal range $8.5-10.6 \mathrm{mg} / \mathrm{dL}$ ). She was taking propranolol, enalapril and simvastatin. Patient's clinical history was uneventful for clinical features related to hypercalcemia. She quit smoking almost twenty years prior; there was no family history of hypercalcemia or urolithiasis.

\section{Investigation}

At baseline, biochemical analyses showed serum calcium, PTH and urinary calcium were above the upper limit of the normal range, while serum $25-\mathrm{OH}$ vitamin $\mathrm{D}$ and phosphorus were below the lowest limit of the normal range (Table 1). Both serum-intact PTH and 25-OH vitamin D were measured by chemiluminescence assay (Diasorin, Stillwater, MN, USA). Serum calcium and phosphorus and all other standard biochemical analyses were performed by a Beckman Coulter DXC 680-I autoanalyzer (Beckman Coulter, Brea, CA, USA). Neck US (performed with ACUSON Antares, $2-13 \mathrm{mHz}$, Siemens, Medical Solution USA) revealed a $4.6 \times 16.1 \times 28.8-\mathrm{mm}$ (volume $1.1 \mathrm{~mL}$ ) hypoechoic, fusiform, solid mass behind the left thyroid lobe (Fig. 1A and Table 1). There were no thyroid nodules or suspicious neck lymph nodes. Due to the location, the US features and clinical data, the mass was strongly suspicious for enlarged parathyroid. Because of the patient's refusal to undergo sestamibi scan and the lack of US, biochemical and clinical features of parathyroid carcinoma, US-FNAB was performed and elevated wPTH $(808 \mathrm{pg} / \mathrm{mL})$ suggestive for parathyroid tissue was assayed in the washing fluid. The cytological examination did not show any atypical cell. Bone mineral density was normal compared to Caucasian standards ( $T$-score of -0.3 and -1.18 at total hip and total spine, respectively and bone mineral content $0.820 \mathrm{~g} / \mathrm{cm}^{2}$ and $0.720 \mathrm{~g} / \mathrm{cm}^{2}$ respectively). The abdominal US revealed some hyperechoic microspots in the kidneys that were suspicious for microlitiasis or microcalcifications. According to the moment guidelines for asymptomatic primary hyperparathyroidism, our patient did not match the criteria for parathyroidectomy, so we recommended a periodic follow-up.

\section{Treatment}

The patient did not undergo medical treatment for PHPT, she only underwent periodic follow-up.

\section{Outcome and follow-up}

At the next visit, after three months, the patient referred she had suffered a slight neck swelling that had resolved

Table 1 Biochemical and US examinations at baseline and during a 96 months (8 years) period of PHPT follow-up in our patient with primary hyperparathyroidism.

\begin{tabular}{l} 
Parameter \\
\hline Serum calcium $(\mathrm{mg} / \mathrm{dL})$ \\
Corrected calcemia* \\
Serum phosphorus $(\mathrm{mg} / \mathrm{dL})$ \\
Urinary calcium $(\mathrm{mg} / 24 \mathrm{~h})$ \\
Serum PTH $(\mathrm{pg} / \mathrm{mL})$ \\
$25-O H$ vitamin D $(\mathrm{ng} / \mathrm{mL})$ \\
Creatinine $(\mathrm{mg} / \mathrm{dL})$ \\
GFR** $\left(\mathrm{mL} / \mathrm{min} / 1.73 \mathrm{~m}^{2}\right)$ \\
Neck US \\
\hline
\end{tabular}

\begin{tabular}{c}
\hline Normal range \\
\hline $8.5-10.6$ \\
- \\
$2.5-5.1$ \\
$100-300$ \\
$15-88$ \\
$30-100$ \\
$0.5-1.2$ \\
$60-90$ \\
\end{tabular}

\begin{tabular}{c}
\hline Baseline \\
\hline 11.3 \\
11.1 \\
2.3 \\
330.2 \\
249.0 \\
21.9 \\
0.8 \\
67.6 \\
$4.6 \times 16.1 \times 28.8 \mathrm{~mm}$ (volume \\
$1.1 \mathrm{~mL}$ ) enlarged parathyroid
\end{tabular}

\begin{tabular}{c}
\hline months after US-FNAB \\
\hline 9.3 \\
9.1 \\
3.0 \\
104.0 \\
90.1 \\
- \\
- \\
No evidence of the \\
enlarged parathyroid
\end{tabular}

\begin{tabular}{c}
\hline 105 months after US-FNAB \\
\hline 9.3 \\
9.1 \\
3.0 \\
102.0 \\
53.1 \\
33.0 \\
0.7 \\
70.1 \\
No evidence of the \\
enlarged parathyroid
\end{tabular}

*Corrected calcemia was calculated using the formula: corrected calcemia $(\mathrm{mg} / \mathrm{dL})=$ total serum calcemia $(\mathrm{mg} / \mathrm{dL})+0.8 \times(4-\mathrm{serum}$ albumin $(\mathrm{g} / \mathrm{dL}))$;

** glomerular filtration rate (GFR) was calculated using the Cockcroft-Gault formula normalized for body surface. 

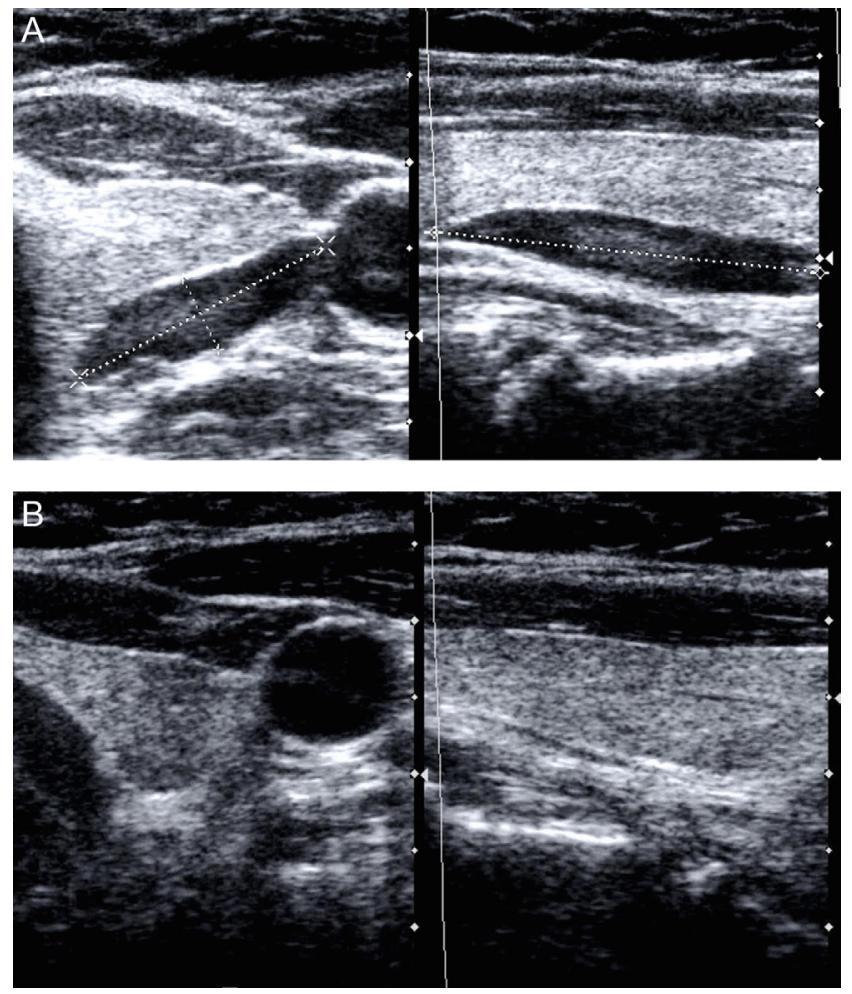

Figure 1

(A) Solid, hypoechoic, fusiform parathyroid (hyperplastic gland/adenoma) localized posterior to the left thyroid lobe at US. (B) Last US of the neck confirming no evidence of the previous abnormal parathyroid gland.

spontaneously in the days after the US-FNAB. Serum calcium, phosphorus, PTH and urinary calcium were all within the normal range (Table 1). During US, the resulting parathyroid lesion disappeared (Fig. 1B and Table 1).

Table 1 lists the results of biochemical and US examinations during the whole follow-up. Biochemical and hormonal parameters remained in the normal range throughout the entire follow-up period of 105 months (Table 1). Even the biannual US evaluations showed no evidence of the previous lesion (Table 1). Neither a magnetic resonance of the neck performed nine months after the US-FNAB showed evidence of hemorrhage or infarction in the previous adenoma localization.

\section{Discussion}

To our knowledge, this is the first reported case of persistent remission of PHPT after US-FNAB performed on a hyperfunctioning enlarged parathyroid resulting in complete disappearance of the lesion and negative biochemical follow-up over a period of 105 months (almost 9 years).
In literature, similar cases have been previously described, but almost all of them concerned cystic parathyroid lesions that relapsed rapidly (7). Only two cases of long-lasting remission of PHPT due to spontaneous infarction of a solid parathyroid adenoma have been described $(8,9)$ : in the first case, parathyroid scans could not localize the parathyroid adenoma for five years (8), but latter the patient probably developed a recurrence (8), while in the second case, the follow-up was just four years long (9).

Spontaneous remission of PHPT can occur rarely. Nylen and coworkers suggested that the pathogenesis of 'autoparathyroidectomy' can be divided into three broad etiologies: (i) autoinfarction (necrosis without hemorrhage), (ii) acute extracapsular hemorrhage and (iii) acute intracapsular hemorrhage (10). However, the etiology of 'autoparathyroidectomy' remains still not clear, especially in clinical cases lacking histological verification. Besides, the time of recurrence depends on the degree of cellular damage (10). In the present case, modest local symptoms and the lack of evidence of the previously documented parathyroid lesion, along with the prolonged uneventful biochemical follow-up, strongly suggest a US-FNAB-induced intracapsular hemorrhage rather than an infarction. A US-FNABinduced intracapsular hemorrhage followed by complete disappearance of the parathyroid lesion might be the consequence of damage involving the entire cell component of the lesion and might explain the complete hormonal and ultrasonographic remission of PHPT. However, in the absence of histological verification, the intracapsular hemorrhage of the enlarged parathyroid remains only a hypothesis, while the causative role of US-FNAB on the disappearance of the parathyroid lesion remains undoubtful.

The results of this case report coupled to the literature review of analogues cases suggest that hyperfunctioning parathyroid benign lesions might be susceptible to both spontaneous and/or FNAB-induced damage. The latter can cause an infarction or hemorrhage with subsequent disappearance of the lesion and remission of the disease, both depending on the degree of cellular damage. This characteristic fragility and susceptibility to necrotic infarction of the parathyroid tissue adenomas might also explain why poor invasive treatments such as highintensity focused US (HIFU), percutaneous ethanol ablation, US-guided radiofrequency and microwave ablation can be effective in obtaining shrinkage with subsequent restoration of PTH and calcium serum levels even though these procedures might have only temporary effects. 
In conclusion, this is the first reported case of complete disappearance of a hyperfunctioning enlarged parathyroid lesion (hyperplastic gland/adenoma) after US-FNAB performed on it with such a long follow-up documenting PHPT complete biochemical and ultrasonographic remission.

In clinical practice, in case of disappearance of the parathyroid lesion and of clinical correlates, a possible recurrence should be considered and adequate clinical and biochemical follow-up remains mandatory for long periods. Accordingly, such lesions, can relapse very frequently due to incomplete damage of the cellular component with subsequent regrowth. However, complete remission of PHPT after invasive procedures is possible as demonstrated by this anecdotal case. Furthermore, these cases can help for better understanding the underlying mechanism of 'autoparathyroidectomy' and can prompt further impulse to the use of less invasive techniques (such as HIFU, alcohol or radiofrequency ablation, etc) in order to avoid surgery when possible.

\section{Declaration of interest}

The authors declare that there is no conflict of interest that could be perceived as prejudicing the impartiality of the research reported.

\section{Funding}

This research did not receive any specific grant from any funding agency in the public, commercial or not-for-profit sector

\section{Patient consent}

The patient provided written informed consent for the publication of all data and pictures of this case report.

\section{Patient's perspective}

We welcome comments from your patient; their own description of their experience may help other patients or clinicians who are dealing with a similar problem. If your patient would like to contribute please ensure they include only relevant personal details. Patients may describe their symptoms, how any tests and treatments affected them and how the problem is now.

\section{Author contribution statement}

E K, E D V, B M and V R designed and drafted the manuscript. V R and $B M$ critically revised the manuscript and data interpretation. E K, E D V, S D V and B M participated to the endocrine assessment and treatment of the patient and data collection. All authors read and approved the final version of the manuscript.

\section{References}

1 Marcocci C \& Cetani F 2011 Clinical practice. Primary hyperparathyroidism. New England Journal of Medicine 365 2389-2397. (https://doi.org/10.1056/NEJMcp1106636)

2 Kunstman JW, Kirsch JD, Mahajan A \& Udelsman R 2013 Parathyroid localization and implications for clinical management. Journal of Clinical Endocrinology and Metabolism 98 902-912. (https:// doi.org/10.1210/jc.2012-3168)

3 Zini M, Attanasio R, Cesareo R, Emmolo I, Frassoldati A, Giannotti L, Guglielmi R, Piovesan A, Procopio M, Scillitani A, et al. 2012 AME position statement: primary hyperparathyroidism in clinical practice. Journal of Endocrinological Investigation 35 2-21. (https://doi. org/10.1007/BF03345413)

4 Kwak JY, Kim EK, Moon HJ, Kim MJ, Ahn SS, Son EJ \& Sohn YM 2009 Parathyroid incidentalomas detected on routine ultrasound-directed fine-needle aspiration biopsy in patients referred for thyroid nodules and the role of parathyroid hormone analysis in the samples. Thyroid 19 743-748. (https://doi.org/10.1089/thy.2008.0263)

5 Cho M, Oweity T, Brandler TC, Fried K \& Levine P 2017 Distinguishing parathyroid and thyroid lesions on ultrasoundguided fine-needle aspiration: a correlation of clinical data, ancillary studies, and molecular analysis. Cancer 125 674-682. (https://doi. org/10.1002/cncy.21888)

6 Bilezikian JP, Brandi ML, Eastell R, Silverberg SJ, Udelsman R, Marcocci C \& Potts JT 2014 Guidelines for the management of asymptomatic primary hyperparathyroidism: summary statement from the fourth international workshop. Journal of Clinical Endocrinology and Metabolism 99 3561-3569. (https://doi.org/10.1210/jc.2014-1413)

7 Maxwell JH, Giroux L, Bunner J \& Duvvuri U 2011 Fine-needle thyroid aspiration-induced hemorrhage of an unsuspected parathyroid adenoma misdiagnosed as a thyroid nodule: remission and relapse of hyperparathyroidism. Thyroid 21 805-808. (https:// doi.org/10.1089/thy.2010.0200)

8 Kovacs KA \& Gay JD 1998 Remission of primary hyperparathyroidism due to spontaneous infarction of a parathyroid adenoma: case report and review of the literature. Medicine $\mathbf{7 7}$ 398-402. (https://doi.org/10.1097/00005792-199811000-00005)

9 Schinner S, Fritzen R, Schott M, Willenberg HS \& Scherbaum WA 2007 Spontaneous remission of primary hyperparathyroidism. Experimental and Clinical Endocrinology and Diabetes 115 619-621. (https://doi.org/10.1055/s-2007-985358)

10 Nylen E, Shah A \& Hall J 1996 Spontaneous remission of primary hyperparathyroidism from parathyroid apoplexy. Journal of Clinical Endocrinology and Metabolism 81 1326-1328. (https://doi. org/10.1210/jcem.81.4.8636326)

Received in final form 24 October 2017 Accepted 2 November 2017 